\title{
Las TIC y la práctica pedagógica, en los docentes de instituciones
}

\section{particulares del Perú}

ICT and pedagogical practice, in teachers from private institutions in

Peru

TIC e prática pedagógica em professores de instituições privadas no

Peru

Tamara Elizabeth Quille Pintado

tamaraquille@upeu.edu.pe

https://orcid.org/0000-0001-9245-2298

Universidad Peruana Unión
Dirma Bernal Altamirano

dirmabernal@upeu.edu.pe

iD https://orcid.org/0000-0001-6222-6214

Universidad Peruana Unión

\author{
Edilberto Cueva Pérez \\ ecuevap@undac.edu.pe \\ iD https://orcid.org/0000-0002-0351-1442 \\ Universidad Nacional Daniel Alcides Carrión
}

Recibido: 24 de junio de 2021

Aceptado: 28 de diciembre 2021

\section{Resumen}

En el estudio se han abordado dos variables: el uso de las TIC y la práctica pedagógica de los docentes, con el objetivo de determinar la relación de ambas variables, en una muestra de 142 docentes, establecida siguiendo el criterio de conveniencia, en el contexto de un enfoque cuantitativo, de tipo descriptivo, correlacional y transversal. Sus resultados mostraron una relación positiva, directa y media de 50.9\%. Se concluye que el uso de las TIC tiene una relación significativa, positiva y directa con la práctica pedagógica de los docentes.

Palabras clave: Uso de las TIC, práctica pedagógica, temporalidad, personal, interpersonal, didáctica. 


\section{Abstract}

The study has addressed two variables: the use of ICT and the pedagogical practice of teachers, with the aim of determining the relationship of both variables, in a sample of 142 teachers, established following the criterion of convenience, in the context of a quantitative, descriptive, correlational and transversal approach. Their results showed a positive, direct and average relationship of $50.9 \%$. It is concluded that the use of ICT has a significant, positive and direct relationship with the pedagogical practice of teachers.

Keywords: Use of ICT, pedagogical practice, temporality, personal, interpersonal, didactic.

\section{Resumo}

O estudo abordou duas variáveis: a utilização das TIC e a prática pedagógica dos professores, de forma a determinar a relação de ambas as variáveis, numa amostra de 142 professores, estabelecida segundo o critério de conveniência, no contexto de uma abordagem quantitativa, descritiva, abordagem correlacional e transversal. Seus resultados mostraram uma relação positiva, direta e média de 50,9\%. Conclui-se que o uso das TIC tem relação significativa, positiva e direta com a prática pedagógica dos professores.

Palavras-chave: Uso das TIC, prática pedagógica, temporalidade, pessoal, interpessoal, didático.

\section{Introducción}

La educación es concebida desde la importancia de la misma, la cual es percibida desde tres ángulos: individual grupal y social (Castellano y Díaz, 2020). La educación, la investigación y la tecnología se encuentran en una perspectiva educativa desde el año 2001, posiblemente con el propósito de mejorar los rendimientos académicos de los estudiantes (Grisales, 2018). Las TIC se han constituido en los recursos determinantes de la educación, muy indispensables para la práctica académica, implica la presencia de una labor diaria de los estudiantes y los docentes, cuyo valor es muy ponderado para el proceso enseñanza aprendizaje (Hernández, Orrego y Quiñones, 2018). El uso de las TIC, según la percepción de los estudiantes y los docentes, genera un acercamiento de la 
escuela y la familia, entendiendo que se ha constituido en una herramienta muy poderosa para el aprendizaje y la investigación (Aparicio, 2018).

En este universo aparecen algunos problemas; por ejemplo, la sociedad en su plenitud, los estudiantes y los profesores experimentarán "algún resentimiento", si no han recibido orientación, preparación, capacitación, dejando un universo lleno de muchos riesgos (Orosco y Pomasunco, 2020).

En pleno siglo XXI, el sistema educativo responde a los cambios exigidos por la llamada era digital, integrando la sociedad, las personas y las instituciones educativas, cuyas herramientas tecnológicas facilitan la educación y el desarrollo del proceso enseñanza aprendizaje (Cueva, 2020). No solamente son necesarias las competencias en la TIC, sino el uso de las mismas y los respectivos enfoques de aprendizaje, con el propósito de generar una correspondencia precisa, objetiva y pertinente a una educación de calidad (Díaz, Almerich, Suárez y Orellana, 2020).

Borgobello, Madolesi, Espinosa y Sartori (2019) realizaron una investigación, en la cual utilizaron una metodología descriptiva, cuya población estuvo constituida por 452 docentes, a quienes aplicaron un cuestionario online sobre características personales y académicas, experiencias, formación y formas mediadas de comunicación con los estudiantes. Los resultados del estudio muestran preferencias y experiencias en el uso de Facebook, WhatsApp y Plataforma virtual. Se llegó a la conclusión de que, por los cuestionamientos colectivos, en trabajos cooperativos y colaborativos la universidad consolidaría su visión institucional más ligada a lo tradicional y conservador, dejando al margen la visión pedagógica más moderna y vinculadas al uso de TIC.

Mamani (2019) presentó un trabajo de investigación sobre la gestión pedagógica y la práctica docente, con el objetivo de determinar la relación de ambas variables, con un enfoque cuantitativo, con diseño de investigación básico, descriptivo, correlacional. La muestra fue de 2 directivos, 36 docentes y 220 estudiantes de la Institución Educativa. Se aplicó dos cuestionarios: uno para cada variable, con 36 ítems para la variable: gestión pedagógica; 23 para la variable: práctica docente. Se concluye que sí existe una relación significativa entre la calidad de la gestión pedagógica y la práctica docente.

Por su parte, Pariona (2019) estudia la gestión pedagógica y la práctica pedagógica, en el ámbito de un enfoque cuantitativo, no experimental, con un diseño descriptivo, 
correlacional, de corte transversal, en una muestra de 40 docentes; para recabar las información se usó la técnica de la encuesta. El estudio en sus conclusiones deja el registro de que no hubo influencia de la gestión educativa sobre la práctica pedagógica. Según Hernández et al. (2020), la práctica pedagógica no ha sido evaluada plenamente en su carácter de diagnóstico y formativo, cuyos estudios son bastantes escasos, debido a su complejidad, cuya debilidad se percibe en la normativa curricular nacional.

Mendoza y Lukis (2020) realizaron un estudio sobre las actitudes de los docentes, respecto del uso de las TIC y la práctica pedagógica, cuyo objetivo fue describir cuáles son las actitudes de los docentes, con la participación de 177 docentes, en un estudio cuantitativo, descriptivo, transeccional, no experimental. Los resultados mostraron actitudes negativas y positivas; además se concluye que es negativa la actitud de los docentes hacia la práctica de las TIC.

La presente investigación responde al objetivo: determinar la relación del uso de las TIC con la práctica pedagógica, en los docentes. En este sentido, se ha realizado un abordaje de las concepciones y algunos aspectos teóricos de las dos variables, con el propósito de tener claridad teórica, práctica y teórica, durante la recepción, análisis e interpretación de los datos obtenidos.

\section{Las TIC}

\section{Conceptos y concepciones diversas}

Según Martínez (2019),

las TIC son un conjunto de técnicas, avances y dispositivos desarrollados que facilitan la productividad en el campo laboral, con determinados usos que ayudan en la facilitación de aprendizaje, ya que proveen herramientas digitales necesarias que permiten la creación de nuevos ambientes en el campo laboral, que conlleva a una mejor en las prácticas periodísticas(p.5).

Son dispositivos digitales, "herramientas más potentes y versátiles" conocidas hasta el momento por la sociedad, potenciales para los docentes y los estudiantes, en el ámbito de la educación y el proceso enseñanza aprendizaje (Cueva, 2020). Son el conjunto de medios, métodos y valores, los cuales generan información y comunicación, permitiendo 
el almacenamiento, el proceso y la transmisión de la información, en forma muy rápida y en cantidades grandes (Mendoza y Lukis, 2020).

\section{Importancia de las TIC}

¿Para qué sirven las TIC, solamente para los docentes, solamente para los estudiantes? La TIC permiten la ejecución de las tareas académicas, aumentan la participación y la iniciativa de los participantes, la relación entre los estudiantes, entre los docentes y los estudiantes, generando una motivación y una actitud positivas (C. Mendoza \& Lukis, 2020).

\section{Dimensiones de las TIC}

\section{Contexto}

La incorporación de las TIC, a la educación se ha convertido en un proceso, cuya implicancia, va mucho más allá de las herramientas tecnológicas que conforman el ambiente educativo, se habla de una construcción didáctica y la manera cómo se pueda construir y consolidar un aprendizaje significativo en base a la tecnología, en estricto pedagógico se habla del uso tecnológico a la educación. (Díaz, 2013, en Hernandez, 2017); han logrado convertirse en instrumentos educativos, capaces de mejorar la calidad educativa del estudiante, revolucionando la forma en que se obtiene, se maneja y se interpreta la información. (Aguilar, 2012, en Hernandez, 2017); el empleo de las TIC en el salón de clase es importante, debido a que ayuda a elevar el desarrollo cognitivo de los estudiantes de una manera dinámica y didáctica, adquieren importancia en la formación docente y no solo en la formación inicial, sino durante toda la vida profesional, porque cada vez más éstas juegan un rol fundamental en el aprendizaje de los alumnos, ya que la información es buscada y encontrada más rápido que dentro de las escuelas (Bautista, 2007, en Cruz et al., 2018).

\section{Nivel de manejo}

Una práctica pedagógica de calidad debe tener acompañamiento de herramientas tecnológicas en el aula, las cuales permitan enriquecer las prácticas pedagógicas (Cortés et al., 2017); en la práctica docente si se requiere integrar las TIC para poder realizar innovaciones educativas, por ello se requieren tanto una infraestructura adecuada en las 
escuelas como una formación en alfabetización digital para los profesores (Fernández \& Pérez, 2018, en Sáenz, 2020).

\section{Ideas, perspectivas y actitudes}

Las creencias de los docentes acerca del valor que aportan las TIC a sus prácticas educativas. Las creencias se conforman a través de experiencias asociadas a situaciones y sucesos personales, los cuales no están abiertos a la evaluación externa o al razonamiento crítico (Nespor, 1987, citado por Coppari \& Bagnoli, 2020).

Las actitudes de los docentes hacia las TIC son: tecnofilia, tecnofobia y crítica. La primera es una actitud plenamente positiva, extremadamente, cuyo proceso de aprendizaje solamente se mejora con el uso de las TIC, se experimenta una total dependencia. Respecto de la segunda, los docentes muestran una actitud negativa, consideran que es una pérdida de tiempo. La actitud crítica significa, por su parte, la presencia de una actitud positiva y mesurada, sometida a una evaluación permanente y crítica, motivando y afianzando el aprendizaje (Mendoza y Lukis, 2020)

\section{Práctica pedagógica}

\section{Conceptos y concepciones}

En el ámbito de la educación sistematizada, se ha construido un universo de conceptos; por ejemplo, práctica pedagógica, práctica docente y práctica de enseñanza; por ejemplo, Ademar \& Rúa (2018) refieren, en este contexto, las prácticas de enseñanza, las cuales replican acciones conscientes y concretas, desarrolladas en el interior de las instituciones educativas, mediante la interacción de las personas participantes: docentes y estudiantes, en el marco de teorías, principios éticos, calidad de exámenes. Por su parte, Salinas (2014), respecto de la práctica docente agrega:

La práctica docente es de carácter social, objetivo e intencional. En ella intervienen los significados, percepciones y acciones de las personas involucradas en el proceso educativo (alumnos, docentes, padres, autoridades, etc.). También intervienen los aspectos político-institucionales, administrativos y normativos, que, en virtud del proyecto educativo de cada país, delimitan el rol del maestro (26). 
Molano et al. (2020) abordan la práctica pedagógica desde la experiencia de los estudiantes, para quienes estas prácticas son formativas y tienen el sentido de herramientas, cuyo propósito es encontrar la mejora continua. Para Mendoza et al. (2020),

la práctica pedagógica es producto de una cultura, de una historia y ante ellas ontológicamente se presenta como una entidad compleja, dinámica, susceptible de cambios, con re- estructuraciones como producto de la acción social y de la misma cultura de la cual forma parte (p. 113).

\section{Importancia}

La práctica pedagógica implica desarrollar la capacidad de articulador, en la experiencia del docente, cuyos procesos pedagógicos no son desarticulados de la dinámicas administrativas; desarrolla las competencias funcionales, la gestión académica, los procesos de planeación, organización (Hernández et al., 2020).

La práctica pedagógica y las experiencias educativas "conllevan al desarrollo de conocimientos, va- lores, actitudes, virtudes, habilidades y destrezas”, en consonancia "con los requerimientos sociales y culturales", "con la participación de todos los actores vinculantes con el proceso educativo" (Mendoza et al., 2020).

Esta práctica implica la realización de la autoevaluación, la coevaluación y la heteroevaluación, constituyéndose en prácticas pedagógicas muy importantes, para la toma de conciencia sobre los conocimientos previos, las metodologías, la calidad de los conocimientos, los recursos; estas herramientas permiten la innovación del proceso enseñanza aprendizaje (Basurto et al., 2021).

\section{Dimensiones}

En el ámbito educativo se precisan algunos conceptos semejantes en sus contenidos y concepciones. Por ejemplo, gestión educativa, práctica docente, práctica pedagógica.

La práctica pedagógica es compleja, multidimensional. Se habla de dimensiones políticas, sociales, culturales, éticas, personal, interpersonal, pedagógica, reveladas mediante las diversas acciones humanas. Las acciones pedagógicas tienen el propósito de orientar la metodología: “participación, investigación, acción y reflexión” (Mayor, 2019). 
El sistema educativo ofrece la gestión educativa en la condición de un componente esencial; cuyas dimensiones son: "pedagógica-curricular, institucional u organizativa, administrativa y social-comunitaria”, cuya práctica docente incluye las dimensiones: "personal, institucional,

interpersonal, social, didáctica y valoral” (Ramírez, 2020). Barolli et al. (2019) considera que el desarrollo profesional de los docentes de ciencias ofrece las dimensiones de análisis, destacando la dimensión pedagógica del oficio docente.

\section{Metodología}

\section{Tipo de investigación}

La presente investigación es de enfoque cuantitativo, no experimental, no se han manipulados las variables; descriptivo, correlacional, transversal. Se describe las características de las variables; se estudia la relación de las mimas; se recaba la información o los datos en un solo momento, haciendo un corte el tiempo de la investigación (Hernández et al., 2014).

\section{Diseño de la investigación}

De acuerdo con Hernández et al. (2014), se realizado un diseño correlacional, mediante el cual se aborda las relaciones de las dos variables y sus respectivas dimensiones: Uso de la TIC y práctica pedagógica, cuyo diseño es el siguiente:

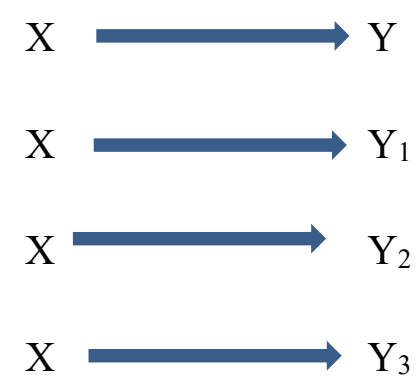

Donde:

$\mathrm{X}=$ Uso de las TIC

$\mathrm{Y}=$ Práctica pedagógica.

$\mathrm{Y}_{1}=$ Práctica pedagógica personal 
$\mathrm{Y}_{2}=$ Práctica pedagógica interpersonal

$\mathrm{Y}_{3}=$ Práctica pedagógica didáctica

\section{Población de la población}

Para Cortés et al. (2017), la población es un conjunto de casos, definido, limitado y accesible, cuyo conjunto formará el referente cuantitativo, para la elección y determinación de la muestra, habiendo cumplido una serie de criterios predeterminados convencionalmente. La población está constituida por 200 maestros de los colegios privados del Perú, de una organización religiosa, la cual funciona en el sur del Perú.

\section{Muestra y muestreo de la investigación}

Los docentes participantes son 142, de los 200. La muestra fue determinada en forma intencional y por conveniencia; se determinó mediante un muestreo no probabilístico, no se aplicaron procedimientos estadísticos; es decir, criterios paramétricos, porque la población es pequeña; la intención fue trabajar con el total de la población; sin embargo, no todos aceptaron su participación; razón por la cual solamente participaron 142 docentes, quienes dieron su consentimiento respectivo, entendiendo que su participación solamente generaría beneficios para la investigación, siendo conscientes de que no les generaría problemas de ningún tipo, menos conflictos ético y morales.

\section{Técnica de recolección de datos}

Se utilizo la técnica denominada encuesta, debido a que el estudio es de enfoque cuantitativo, cuyos instrumentos, correspondientes a las dos variables, fueron aplicados a los 142 docentes, quienes dieron su consentimiento pertinente; además participaron voluntariamente. Los datos fueron recabados mediante la aplicación del instrumento, en forma virtual; luego los datos fueron procesados en Microsoft Excel y el programa estadístico SPSS V22.

\section{Instrumentos de recolección de datos}

Para la recolección de datos se utilizó un instrumento, el cual contiene dos partes: una para medir cada variable; es decir, una para el uso de las TIC y otra para evaluar la práctica pedagógica. El autor del instrumento es Jorge Príncipe Villanueva (Principe, 2017), quien lo construyó y lo sometió a la validación respectiva, mediante juicio de 
expertos; quienes validaron el instrumento fueron: Juliana Chumbe Muños, Walter A. Santibáñez Bernardo, Modesto Paucar Serpa, Esmirio Erotides Castillo Gonzales. El instrumento, en la parte de la medición de las TIC, tiene 21 ítems, distribuidos en tres dimensiones: "contexto relacionado con las tecnologías", "manejo y aplicación en la educación" y "idea y perspectivas sobre las TIC". Para evaluar todos ítems del instrumento, en sus dos partes, se utilizó la escala tipo Likert, con las denominaciones: Bajo (1), Moderado (2) y Alto (3). La primera variable tiene 6 ítems (1-6); la segunda variable, 8 (7-14); la tercera, 7 (15-21).

La segunda parte del instrumento, referida a la práctica pedagógica, el instrumento contiene 25 ítems, distribuidos en tres dimensiones: práctica pedagógica personal, práctica pedagógica interpersonal y práctica pedagógica didáctica. La primera dimensión tiene 11 ítems (1-11); la segunda, 4 (12-15); la tercera, 10 (16-25). El instrumento fue aplicado en la presente investigación, con la autorización escrita del autor.

\section{Confiabilidad del instrumento}

Tabla 1

Prueba de confiabilidad

\begin{tabular}{rr}
\hline Alfa de Cronbach & \multicolumn{2}{l}{ N de elementos } \\
& .949 \\
\end{tabular}

La confiabilidad del instrumento es de $94.9 \%$, cuyo porcentaje significa que el cuestionario usado se caracteriza, porque es muy confiable estadísticamente.

Operación de variables

\begin{tabular}{|l|l|}
\hline Variables & Dimensiones \\
\hline \multirow{2}{*}{ Uso de las TICS } & Contexto \\
\cline { 2 - 2 } & Nivel de manejo \\
\cline { 2 - 2 } & Ideas y perspectiva \\
\hline \multirow{2}{*}{ Práctica docente } & Personal \\
\cline { 2 - 2 } & Interpersonal \\
\cline { 2 - 2 } & Didáctica \\
\hline
\end{tabular}




\section{Resultados}

Los datos y los resultados de la investigación fueron procesados, organizados y sistematizados, con el propósito de generar análisis, interpretaciones y discusiones. En este contexto, se presentan los análisis de los datos demográficos y las pruebas de la hipótesis.

\section{Análisis de los datos demográficos}

Tabla 2.

Información demográfica

\begin{tabular}{|c|c|c|c|}
\hline Variables & Frecuencia & Porcentaje & $\begin{array}{l}\text { Porcentaje } \\
\text { acumulado }\end{array}$ \\
\hline \multicolumn{4}{|l|}{ Género } \\
\hline Masculino & 43 & 30.3 & 30.3 \\
\hline Femenino & 99 & 69.7 & 100.0 \\
\hline Total & 142 & 100.0 & \\
\hline $\begin{array}{l}\text { Región de la } \\
\text { institución } \\
\text { educativa }\end{array}$ & & & \\
\hline \multicolumn{4}{|l|}{ Peruano } \\
\hline Misión Peruana & 45 & 31.7 & 62.7 \\
\hline \multicolumn{4}{|l|}{ Central Sur } \\
\hline Asociación & 50 & 35.2 & 97.9 \\
\hline \multicolumn{4}{|l|}{ Educativa Nor } \\
\hline \multicolumn{4}{|l|}{ Oriental } \\
\hline Asociación Peruana & 3 & 2.1 & 100.0 \\
\hline Central Este & & & \\
\hline
\end{tabular}


Total

Nivel laboral

Inicial

Primario

Secundario

Total
142 11

72

59

142
100.0

Los datos sociodemográficos que se analizaron en el presente estudio, fueron: el género, la región de la institución educativa del participante y el nivel laboral del mismo. El $69.7 \%$ de participantes corresponde al género femenino y el $30.3 \%$ al género masculino. Las regiones de ubicación de la institución educativa, a la cual están adscritos los participantes, fueron cuatro: Misión Oriente Peruano, Misión Peruana Central Sur, Asociación Educativa Nor Oriental y Asociación Peruana Central Este, cuyos porcentajes respectivos fueron: $31.0,31.7,35.2$ y 2.1. Respecto del nivel, en el cual laboran los participantes. Del nivel inicial participaron el 7.7\%, del nivel primario el 50.7\% y del nivel secundario el $41.5 \%$.

\section{Prueba de hipótesis}

Prueba de la normalidad de las variables

Tabla 3.

Normalidad de las variables

\section{Kolmogorov-Smirnov ${ }^{\mathrm{a}}$}

Estadístico gl $\quad \mathrm{g}$ valor.

\begin{tabular}{lccc}
\hline Personal & .251 & 142 & .000 \\
Interpersonal & .318 & 142 & .000 \\
Didáctica & .175 & 142 & .000
\end{tabular}


Uso de las

.088

142

.010

TICs

Práctica

.000

pedagógica

La normalidad se realiza y se analiza previamente al análisis de correlación; determina que todas las variables estudiadas presentan una distribución normal, porque el valor más alto de p es 0.010 y es menor que 0.05 . Por lo mencionado, el análisis correlacional se hizo a través de coeficiente de correlación de Rho Spearman.

\section{Relación del uso de las TIC y la práctica pedagógica personal}

Tabla 4.

Relación del uso de las TIC y la práctica pedagógica personal

\section{Personal}

Rho Spearman $\quad$ p valor N

Uso de las

TICs

, $458^{* *} \quad .000$

142

TICs

Después del análisis del coeficiente de correlación de Rho Spearman, se encontró una relación de $45.8 \%$, mostrando una relación de fuerza media; por otro lado, se encontró que la relación existente es positiva; es decir, mientras el uso de las TIC se promueva, la práctica pedagógica personal aumentará. Por último, se encontró que esta relación es significativa, ya que el p valor es de $0.000(\mathrm{p}<0.05)$.

\section{Relación del uso de las TIC y la práctica pedagógica interpersonal}

Tabla 5 .

Relación del uso de las TIC y la práctica pedagógica interpersonal 


\begin{tabular}{lccc}
\hline & \multicolumn{2}{c}{ Interpersonal } \\
& Rho Spearman & $\mathrm{p}$ valor & $\mathrm{N}$ \\
\hline $\begin{array}{c}\text { Uso de las } \\
\text { TICs }\end{array}$ &, $221^{* *}$ & .008 & 142 \\
& & & \\
\hline
\end{tabular}

Realizado el análisis del coeficiente de correlación de Rho Spearman, se percibe una relación de $22.1 \%$, dejando la evidencia de una relación de fuerza baja; por otro lado, se observó que la relación es positiva; dicho de otro modo, mientras el uso de las TIC más se promueva, la práctica pedagógica interpersonal aumentará. También se encontró que esta relación es significativa, ya que el p valor es de $0.008(\mathrm{p}<0.05)$.

\section{Relación del uso de las TIC y la práctica pedagógica didáctica}

Tabla 6.

Relación del uso de las TIC y la práctica pedagógica didáctica

\section{Didáctica}

Rho Spearman $\quad$ p valor N

\begin{tabular}{cccc}
\hline Uso de las &, $524^{* *}$ & .000 & 142 \\
TICs & &
\end{tabular}

Concluido el análisis del coeficiente de correlación de Rho Spearman, se precisó una relación de 52.4\%, mostrando una relación de fuerza media; asimismo, se determinó que la relación existente es positiva; vale decir, si más se promueve el uso de las TIC, entonces la práctica pedagógica didáctica aumentará. Por otro lado, se encontró que esta relación es significativa, porque el $\mathrm{p}$ valor es de $0.000(\mathrm{p}<0.05)$.

\section{Relación del uso de las TIC y la práctica pedagógica}

Tabla 7.

Relación del uso de las TIC y la práctica pedagógica 


\begin{tabular}{lccc}
\hline \multicolumn{2}{c}{ Práctica pedagógica } \\
& Rho Spearman & $\mathrm{p}$ valor & $\mathrm{N}$ \\
\hline $\begin{array}{c}\text { Uso de las } \\
\text { TICs }\end{array}$ &, $509^{* *}$ & .000 & 142 \\
& & & \\
\hline
\end{tabular}

Después de la aplicación del coeficiente de correlación de Rho Spearman, se encontró una relación de 50.9\%, mostrando una relación de fuerza media; por otro lado, se observó que la relación existente es positiva; es decir, mientras el uso de las TIC más se promueva, la práctica pedagógica aumentará. Además, se precisó que esta relación es significativa, ya que el $p$ valor es de $0.000(p<0.05)$.

\section{Discusión}

El presente estudio abordó las variables: uso de las TIC y la práctica pedagógica de los docentes, realizado con la participación de 142 docentes, procedentes de varias regiones del Perú, adscritos a una organización educativa particular. La variable: uso de las TIC fue estudiada en sus tres dimensiones: contexto, nivel de manejo, ideas y perspectivas. La práctica pedagógica, en sus tres dimensiones: personal, interpersonal y didáctica. El uso de las TIC y la práctica pedagógica tienen una relación de 50.9\%; por otro lado, el uso de las TIC y la práctica pedagógica personal tiene una relación de $45.8 \%$; el uso de las TIC y la práctica pedagógica interpersonal, una relación de 22.1\%. El uso de las TIC y la práctica pedagógica didáctica, una relación de $52.4 \%$. Según los datos del estudio, se confirma que la relación de las variables estudiadas es positiva, aunque no muy significativa, porque la relación está por debajo de la media, con excepción de la práctica pedagógica, cuyo porcentajes 50.9\%; también de la práctica pedagógica didáctica, cuya relación es de $52.4 \%$; en cambio, las demás relaciones quedan ubicadas por debajo de la media; por ejemplo, la relación con la práctica pedagógica personal (45.8\%) y la práctica pedagógica interpersonal (22.1\%).

Por su parte, Principe (2017) realizó su estudio, años anteriores al presente, sirviendo de base para esta investigación, exactamente con las mismas variables y las mismas dimensiones, cuyo estudio fe realizado solamente en 25 profesores, del nivel de secundaria, en una sola institución educativa; a diferencia del presente, realizado en 142 
y en los tres noveles. El estudio consultado entrega un nivel moderado de $48 \%$ en el uso de las TIC, también un nivel moderado en la práctica pedagógica. La relación de las dos variables es positiva, con una correlación lineal de $83.6 \%$.

Sobre la importancia del uso de las TIC, Rendón y Jarvio (2020) confirman que, mediante su estudio, las tecnologías promueven la lectura en las bibliotecas públicas; para Cueva (2020) se constituyen "herramientas más potentes y versátiles que la sociedad haya conocido hasta el momento" (p. 341), en el contexto de la educación y la crisis. Mejoran la enseñanza de las matemáticas, generando muchos retos y grandes perspectivas (Grisales, 2018), también las competencias, las actitudes, las habilidades sociales y creativas, relacionadas con las TIC (Fernández et al., 2020; Flores et al., 2016; García y Gómez, 2020).

Por otro lado, el uso de las TIC se hace trascendente; mejora los aprendizajes (Fernández, 2021; Badillo y Iguarán, 2020; Díaz et al., 2020); produce la alfabetización digital de los docentes (Coppari \& Bagnoli, 2020), fortalece las prácticas pedagógicas de los docentes (Borgobello et al., 2019).

\section{Conclusiones}

Luego de concluir todos los procedimientos estadísticos y los análisis teóricos, se concluye que el uso de las TIC tiene una relación muy significativa, directa y positiva con la práctica pedagógica de los docentes, cuyas prácticas son totalmente fortalecidas, generando beneficios para los procesos de enseñanza aprendizaje de los estudiantes.

Por otro lado, el uso de las TIC también tiene una relación positiva, significativa y directa con la práctica pedagógica personal de los docentes; vale decir, el uso referido mejora, fortalece las prácticas individuales de los docentes.

El uso de la TIC, por su parte, permite reforzar la práctica pedagógica, en su dimensión interpersonal; generando crecimiento y desarrollo de los docentes, en su relación pedagógica con los demás, sus colegas, quienes tiene parte ponderativa en el proceso enseñanza aprendizaje.

Los docentes mejoran y fortalecen su práctica pedagógica, en su dimensión didáctica. Es decir, el uso de las TIC determina el crecimiento y desarrollo de los docentes, generando experiencias, virtudes, actitudes, aptitudes, habilidades sociales, creativas e 
instrumentales, en el sentido de beneficiar el proceso enseñanza aprendizaje, por efecto del dominio de los recursos, los medios, los materiales y las metodologías didácticas.

\section{Referencias}

Ademar, H., \& Rúa, A. (2018). Dimensiones involucradas en el estudio de las prácticas de enseñanza. Educación y Humanismo, 20(34), 140-155. https://doi.org/http://dx.doi.org/10.17081/eduhum.20.34.2861

Aparicio, O. Y. (2018). El uso educativo de las TIC. Revista Interamericana de Investigación, Educación y Pedagogía, 2015, 211-227. https://doi.org/DOI https://doi.org/10.15332/s1657-107X.2019.0001.02

Badillo, V. T., \& Iguarán, A. M. (2020). Uso de las TIC en la enseñanza-aprendizaje de la comprensión lectora en niños autistas. Praxis, 16(1), 55-63. https://doi.org/10.21676/23897856.3406

Barolli, E., Nascimento, W. E., Oliveira, J. de, \& Villani, A. (2019). Desarrollo profesional de profesores de ciencias: dimensiones de análisis. Revista Electrónica de Enseñanza de Las Ciencias, 18(1), 173-197. http://revistas.educacioneditora.net/index.php/REEC/article/view/368/37

Basurto, S. T., Moreira, J. A., Velásquez, A. N., \& Rodríguez, M. (2021). Autoevaluación, Coevaluación y Heteroevaluación como enfoque innovador en la práctica pedagógica y su efecto en el proceso de enseñanza-aprendizaje. Pol. Con., 6(3), 828-845. https://doi.org/10.23857/pc.v6i3.2408

Borgobello, A., Madolesi, M., Espinosa, A., \& Sartori, M. (2019). Uso de TIC en prácticas pedagógicas de docentes de la Facultad de Psicología de una universidad pública argentina. Revista de Psicología, 37(1), 279-317. https://doi.org/https://doi.org/10.18800/psico.201901.010

Castellano, N., \& Díaz, B. (2020). Calidad de la práctica pedagógica del docente universitario. Negotium, 46, 42-52. https://doi.org/http://doi.org/10.5281/zenodo.4767693

Coppari, N., \& Bagnoli, L. (2020). Alfabetización Digital de Docentes: Análisis Teórico y Propuesta de Evaluación Piloto. Eureka, 17(1), 112-140. 
https://psicoeureka.com.py/sites/default/files/articulos/eureka-17-1-15.pdf

Cortés, S. M., Vargas, T., \& Neira, J. A. (2017). Uso de las TIC en la práctica pedagógica. Tecnología, Investigación y Academia, 5(1), 46-56.

https://revistas.udistrital.edu.co/index.php/tia/article/view/11128/pdf

Cruz, M. A., Pozo, M. A., Aushay, H. R., \& Arias, A. D. (2018). Las Tecnologías de la Información y la Comunicación (TIC) como forma investigativa interdisciplinaria con un enfoque intercultural para el proceso de formación estudiantil. E-Ciencias de La Información, 9(1), 1-15.

https://doi.org/https://doi.org/10.15517/eci.v1i1.33052

Cueva, D. A. (2020). La tecnología educativa en tiempos de crisis. Revista Conrado, 16(74), 341-348. http://scielo.sld.cu/pdf/rc/v16n74/1990-8644-rc-16-74-341.pdf

Díaz, I, Almerich, G., Suárez, J., \& Orellana, N. (2020). La relación entre las competencias TIC, el uso de las TIC y los enfoques de aprendizaje en alumnado universitario de educación. Revista de Investigacion Educativa, 38(2), 549-566. https://doi.org/http://dx.doi.org/10.6018/rie.409371

Díaz, Isabel, Almerich, G., Suárez, J., \& Orellana, N. (2020). La relación entre las competencias TIC, el uso de las TIC y los enfoques de aprendizaje en alumnado universitario de educación. Revista de Investigación Educativa, 38(2), 549-566. https://doi.org/http://dx.doi.org/10.6018/rie.409371

Fernández, C., Fernández, M. C., Cebreiro, B., Soto, J., Martínez, A. E., \& Casal, L. (2020). Competencias y actitudes para el uso de las TIC de los estudiantes del grado de maestro de Galicia. Publicaciones, 50(1), 103-120. https://doi.org/10.30827/PUBLICACIONES.V50I1.11526

Fernández, J. L. (2021). El uso de las TIC como paliativo de las dificultades del aprendizaje en las ciencias sociales. Digital Education Review, 39, 213-237. https://doi.org/10.1344/DER.2021.39.213-237

Flores, E., Garcia, M. L., Calsina, W. C., \& Yapuchura, A. (2016). Las Habilidades sociales y la comunicación interpersonal de los estudiantes de la Universidad Nacional del Altiplano, Puno. Cominicacción, 7(2), 05-14. http://www.scielo.org.pe/scielo.php?script=sci_arttext\&pid=S2219- 
$71682016000200001 \& \operatorname{lng}=\mathrm{es} \& n r m=\mathrm{iso} \& \operatorname{lng}=\mathrm{es}$

García, C., \& Gómez, J. (2020). Desarrollo de habilidades creativas de los estudiantes como consecuencia del uso de herramientas TIC. Informador Técnico, 84(2), 133154. https://doi.org/10.23850/22565035.2547

Grisales, A. M. (2018). Uso de recursos TIC en la enseñanza de las matemáticas: retos y perspectivas. Entramado, 14(2), 198-214. https://doi.org/10.18041/19003803/entramado.2.4751

Hernández, C. A., Gamboa, A. A., \& Avendaño, W. R. (2020). Evaluación de carácter diagnóstico formativa en maestros de Ciencias Naturales: análisis desde la reflexión y planeación de la práctica pedagógica. Revista ESPACIOS, 41(33), 200 211. http://es.revistaespacios.com/a20v41n33/20413317.html

Hernández, R., Fernández, C., \& Baptista, P. (2014). Metodología de la investigación (Sexta aedi). McGraw Hill Interamericana Editores, S.A. de C.V. https://doi.org/10.1017/CBO9781107415324.004

Hernandez, R. M. (2017). Impacto de las TIC en la educación: Retos y Perspectivas. 5(1), 325. https://doi.org/10.20511/pyr2017.v5n1.149

Hernández, R. M., Orrego, R., \& Quiñones, S. (2018). Nuevas formas de aprender: La formación docente frente al uso de las TIC. Propósitos y Representaciones, 6(2), 671-701. https://doi.org/10.20511/pyr2018.v6n2.248

Mamani, H. (2019). La calidad de la gestión pedagógica y su relación con la práctica docente en el nivel primario de la Institución Educativa $N^{\circ} 70005$ “Corazón de Jesús” Puno-2017 [Tesis de maestría, Universidad Nacional del Altiplano]. http://repositorio.unap.edu.pe/handle/UNAP/11000

Martínez, R. A. (2019). El impacto de las TICs en las prácticas periodísticas en la actualidad [Tesis, Universidad Jaime Bausate y Meza]. http://repositorio.bausate.edu.pe/bitstream/handle/bausate/201/Martínez-VegaRenato-Alonso.pdf? sequence $=1 \&$ isAllowed $=\mathrm{y}$

Mayor, D. (2019). Dimensiones pedagógicas que configuran las prácticas de aprendizaje-servicio. Revista Páginas de Educación, 12(2), 23-42. 
https://doi.org/https://doi.org/10.22235/pe.v12i2.1834

Mendoza, C., \& Lukis, K. (2020). Actitudes de los Docentes en el Uso de las

Tecnologías de la Información y Comunicación (TIC) en la Práctica Pedagógica de una Institución de Educación Superior Privada de la Ciudad de Lima, 2019

[Tesis de maestría, Universidad Tecnológica del Perú].

https://repositorio.utp.edu.pe/handle/20.500.12867/3193?show=full

Mendoza, D. J., Flores, E. M., Revilla, L. S., Cejas, M. F., \& Navarro, M. (2020).

Práctica pedagógica de la educación ecuatoriana en el siglo XXI. EDETANIA, 57, 111-141. https://doi.org/10.46583/edetania

Molano, N. J., Jiménez, G. H., \& Molano, D. X. (2020). La práctica pedagógica de los estudiantes de Educación Física con niños preescolares. Infancias Imágenes, 19(2), 1-24. https://doi.org/10.14483/16579089.14917

Orosco, J. R., \& Pomasunco, R. (2020). Adolescentes frente a los riesgos en el uso de las TIC. Investigación Educativa, 22, e 17, 1-13.

https://doi.org/https://doi.org/10.24320/redie.2020.22.e17.2298

Pariona, K. P. (2019). La Gestión Educativa y la Práctica Docente en la Institución Educativa Integrada Sagrado Corazón de Jesús, San Ramón- ChanchamayoJunín, 2017 [Tesis de maestro, Universidad Nacional de Educación Enrique Guzmán y Valle]. https://repositorio.une.edu.pe/handle/UNE/4308

Principe, J. (2017). El uso de las TICs y la práctica pedagógica de los docentes de nivel secundaria de la Institución Educativa José Gálvez Barrenechea, distrito de Uchiza, región San Martín, 2016 [Tesis, Universidad César Vallejo]. https://repositorio.ucv.edu.pe/bitstream/handle/20.500.12692/27666/B_Principe_V J.pdf?sequence $=4 \&$ is Allowed $=\mathrm{y}$

Ramírez, M. A. (2020). Gestión educativa y práctica docente: reflexiones sobre la dimensión investigativa. Ciencia y Educación, 1(2), 48-65. https://doi.org/https://doi.org/10.48169/Ecuatesis/0102202012

Rendón, S., \& Jarvio, A. (2020). El uso de las TIC para promover la lectura en bibliotecas públicas con la intervención de los bibliotecarios. Investigación Bibliotecológica: Archivonomía, Bibliotecología e Información, 34(83), 129. 
Sáenz, F. D. (2020). Percepciones de docentes sobre competencias digitales: caso de una red de colegios vinculados al Instituto de Informática de una univerdidad privada. [Tessis de magíster, Pontificia Universidad Católica del Perú]. http://hdl.handle.net/20.500.12404/17918

Salinas, E. V. (2014). La calidad de la gestión pedagógica y su relación con la práctica docente en el nivel secundaria de las Institución Educativa Policía Nacional del Perú “Juan Linares Rojas”, Oquendo, Callao-2013 [Tesis de magíster, Universidad Nacional Mayor de San Marcos]. https://cybertesis.unmsm.edu.pe/bitstream/handle/20.500.12672/4240/Salinas_ee.p $\mathrm{df}$ ? sequence $=1$ \&isAllowed $=\mathrm{y}$ 